\title{
Retracted Article: MRI Tracking of the Fate of Intravascularly Injected and SPIO-Labeled Rat Mesenchymal Stem Cells in the Livers of Rats with Hepatic Fibrosis
}

\author{
Zhaofeng Tang $\cdot$ Nan Lin $\cdot$ Heping Fang $\cdot$ \\ Kangshun Zhu $\cdot$ Yong Liu $\cdot$ Yuesi Zhong • \\ Jizhong Lin $\cdot$ Zhuang Kang $\cdot$ Ruiyun Xu
}

Received: 24 March 2009/Accepted: 9 July 2009/Published online: 11 August 2009

(C) Springer Science+Business Media, LLC 2010

This article was published on OnlineFirst, but is withdrawn due to duplication in part of a prior publication.

Z. Tang $\cdot$ N. Lin $\cdot$ H. Fang $\cdot$ Y. Zhong $\cdot$ J. Lin $\cdot$ R. Xu ( $($ )

Department of Hepatobiliary Surgery, The Third Affiliated

Hospital, Sun Yat-sen University, 510630 Guangzhou,

Guangdong Province, China

e-mail: linnancn@gmail.com

\section{K. Zhu $\cdot$ Z. Kang}

Department of Radiology, The Third Affiliated Hospital, Sun Yat-sen University, 510630 Guangzhou, Guangdong

Province, China

Y. Liu

Department of Pathology, The Third Affiliated Hospital, Sun Yat-sen University, 510630 Guangzhou, Guangdong Province, China 